\title{
Novel Global Asymptotic Stability Criteria for Delayed Cellular Neural Networks
}

\author{
Shengyuan Xu, James Lam, Senior Member, IEEE, Daniel W. C. Ho, Member, IEEE, and Yun Zou
}

\begin{abstract}
This brief provides improved conditions for the existence of a unique equilibrium point and its global asymptotic stability of cellular neural networks with time delay. Both delay-dependent and delay-independent conditions are obtained by using more general Lyapunov-Krasovskii functionals. These conditions are expressed in terms of linear matrix inequalities, which can be checked easily by recently developed standard algorithms. Examples are provided to demonstrate the reduced conservatism of the proposed criteria by numerically comparing with those reported recently in the literature.
\end{abstract}

Index Terms-Cellular neural networks (CNNs), global asymptotic stability, linear matrix inequality (LMI), time-delay systems.

\section{INTRODUCTION}

C ELLULAR NEURAL networks (CNNs), which were introduced in [8], have been a subject of intense research activities in the literature over the past years and have found extensive applications in image processing, pattern recognition and classification, solving nonlinear algebraic equations, and other areas [7], [11]. It has been shown that such applications rely on the analysis of the dynamical behavior of CNNs. Since stability is one of the most important issues related to such behavior, the study of the stability problem of CNNs has received much attention in recent years and a great number of results on this issue have been reported; see, e.g., [10], [14], and the references therein.

On the other hand, time delays are often encountered in various practical systems such as chemical processes, long transmission lines in pneumatic systems, biological and neural networks [3], [12]. The existence of time delays may lead to oscillation, divergence or instability in neural networks due to finite speed of information processing. Therefore, many researchers have investigated the problem of stability analysis for neural networks with time delays. For example, the global asymptotic stability of delayed CNNs with bounded and nonmonotonic activation functions were studied in [2] and [5], respectively, where several sufficient conditions were given via

Manuscript received November 19, 2003; revised November 4, 2004. This work was supported by the Research Grants Council under GrantHKU $7127 / 02$ P, by the Foundation for the Author of National Excellent Doctoral Dissertation, China, under Grant 200240, by the National Natural Science Foundation, China, under Grant 60304001 and Grant 60074007, and by the Fok Ying Tung Education Foundation under Grant 91061. This paper was recommended by Associate Editor M. Gilli.

$\mathrm{S}$. Xu and Y. Zou are with the Department of Automation, Nanjing University of Science and Technology, Nanjing 210094, China.

J. Lam is with the Department of Mechanical Engineering, University of Hong Kong, Hong Kong (e-mail: james.lam@hku.hk).

D. W. C. Ho is with the Department of Mathematics, City University of Hong Kong, Hong Kong.

Digital Object Identifier 10.1109/TCSII.2005.849000 different approaches. These results were extended to a more general class of CNNs with delays in [6], [18]. Recently, a linear matrix inequality (LMI) approach to the stability analysis of delayed CNNs was introduced in [13], in which some sufficient conditions were presented. The results in [13] were further improved in [1] by using different Lyapunov-Krasovskii functionals. It is noted that all the above mentioned asymptotic stability results are delay-independent; that is, they do not include any information on the size of delays. It is known that delay-dependent stability conditions, which employ the information on the size of delays, are generally less conservative than delay-independent ones especially when the size of the delay is small [12], [16]. Considering this, a delay-dependent asymptotic stability condition for symmetric delayed CNNs was proposed in [9]. When the $\mathrm{CNN}$ is not symmetric, the delay-dependent results can be found in [17].

In this paper, attention is focused on the derivation of improved conditions for the existence of a unique equilibrium point and its global asymptotic stability of CNNs with time-invariant delay. By constructing more general Lyapunov-Krasovskii functionals, less conservative stability conditions are established. These conditions are expressed in terms of LMIs. It is worth pointing out that the LMI condition can be checked numerically very efficiently by resorting to recently developed interior-point methods, and no tuning of parameters will be involved [4]. Both delay-dependent and delay-independent results are given. Examples are provided to demonstrate the less conservatism of the proposed criteria.

Notation: Throughout this brief, for real symmetric matrices $X$ and $Y$, the notation $X \geq Y$ (respectively, $X>Y$ ) means that the matrix $X-Y$ is positive semi-definite (respectively, positive definite). The superscript " $T$ " represents the transpose. The notation $|\cdot|$ refers to the Euclidean vector norm. We use $\lambda_{\min }(\cdot)$ to denote the minimum eigenvalue of a real symmetric matrix. Matrices, if not explicitly stated, are assumed to have compatible dimensions.

\section{MAIN Results}

Consider a continuous-time $\mathrm{CNN}$ with a constant delay described by the following nonlinear retarded functional differential state equation:

$$
\dot{u}(t)=-A u(t)+W_{0} g(u(t))+W_{1} g(u(t-\tau))+\mathcal{I}
$$

where

$$
u(t)=\left[\begin{array}{llll}
u_{1}(t) & u_{2}(t) & \cdots & u_{n}(t)
\end{array}\right]^{T}
$$


is the state vector

$$
g(u(t))=\left[\begin{array}{llll}
g_{1}\left(u_{1}(t)\right) & g_{2}\left(u_{2}(t)\right) & \cdots & g_{n}\left(u_{n}(t)\right)
\end{array}\right]^{T}
$$

is the neuron activation function with $g(0)=0, A=$ $\operatorname{diag}\left(a_{1}, a_{2}, \ldots, a_{n}\right)>0, W_{0}$ and $W_{1}$ are the interconnection matrices representing the weighting coefficients of the neurons, $\mathcal{I}=\left[\begin{array}{llll}\mathcal{I}_{1} & \mathcal{I}_{2} & \cdots & \mathcal{I}_{n}\end{array}\right]^{T}$ is a constant vector representing the bias. The scalar $\tau>0$ is a constant delay of the system.

The following assumption will be made throughout the paper.

Assumption 1: [6] The activation function $g(u)$ is nondecreasing, bounded and globally Lipschitz; that is

$$
0 \leq \frac{g_{i}\left(\xi_{1}\right)-g_{i}\left(\xi_{2}\right)}{\xi_{1}-\xi_{2}} \leq \sigma_{i}, \quad i=1,2, \ldots, n .
$$

Then, by [6], it can be seen that there exists an equilibrium for (1). For the sake of simplicity in the stability analysis of (1), we make the following transformation to (1):

$$
x(t)=u(t)-u^{*}
$$

where

$$
u^{*}=\left[\begin{array}{llll}
u_{1}^{*} & u_{2}^{*} & \cdots & u_{n}^{*}
\end{array}\right]^{T}
$$

is an equilibrium point of system (1). Under the transformation of (3), it is easy to see that (1) becomes

$$
\dot{x}(t)=-A x(t)+W_{0} f(x(t))+W_{1} f(x(t-\tau))
$$

where

$$
x(t)=\left[\begin{array}{llll}
x_{1}(t) & x_{2}(t) & \cdots & x_{n}(t)
\end{array}\right]^{T}
$$

is the state vector of the transformed system, and

$$
f(x(t))=\left[\begin{array}{llll}
f_{1}\left(x_{1}(t)\right) & f_{2}\left(x_{2}(t)\right) & \cdots & f_{n}\left(x_{n}(t)\right)
\end{array}\right]^{T}
$$

with

$$
f_{i}\left(x_{i}(t)\right)=g_{i}\left(x_{i}(t)+u_{i}^{*}\right)-g_{i}\left(u_{i}^{*}\right), \quad i=1,2, \ldots, n
$$

and $f_{i}(0)=0$, for $i=1,2, \ldots, n$. It is noted that $f_{i}(\cdot)$ satisfies (2); that is

$$
0 \leq \frac{f_{i}\left(\xi_{1}\right)-f_{i}\left(\xi_{2}\right)}{\xi_{1}-\xi_{2}} \leq \sigma_{i}, \quad i=1,2, \ldots, n .
$$

Before presenting the main results, we give the following lemma, which will be used in the sequel.

Lemma 1: [15] Let $\mathcal{D}, \mathcal{S}$ and $\mathcal{P}$ be real matrices of appropriate dimensions with $\mathcal{P}>0$. Then, for any vectors $x, y$ with appropriate dimensions

$$
2 x^{T} \mathcal{D} \mathcal{S} y \leq x^{T} \mathcal{D} \mathcal{P} \mathcal{D}^{T} x+y^{T} \mathcal{S}^{T} P^{-1} \mathcal{S} y .
$$

Now, we are in a position to present a new asymptotic stability condition for system (4), which is independent of the size of the delay.

Theorem 1: The origin of the delayed CNN in (4) is the unique equilibrium point and it is globally asymptotically stable for all delay $\tau>0$ if there exist matrices $P>0, Q>0, Z>0$ and two diagonal matrices $S>0$ and $H>0$ such that the following LMI holds:

$$
\left[\begin{array}{cccc}
-P A-A P & P W_{1} & P W_{0}+H \Sigma & 0 \\
W_{1}^{T} P & W_{1}^{T} Z W_{1}-Q & 0 & 0 \\
W_{0}^{T} P+\Sigma H & 0 & U & S \\
0 & 0 & S & -Z
\end{array}\right]<0
$$

where

$$
\begin{aligned}
& \Sigma=\operatorname{diag}\left(\sigma_{1}, \sigma_{2}, \ldots, \sigma_{n}\right) \\
& U=Q-2 H-2 S A \Sigma^{-1}+S W_{0}+W_{0}^{T} S .
\end{aligned}
$$

Proof: We prove this theorem in two steps. Firstly, we show the uniqueness of the equilibrium point by contradiction. Let $\bar{x}$ be the equilibrium point of the delayed CNN in (4). Then, we have

$$
-A \bar{x}+\left(W_{0}+W_{1}\right) f(\bar{x})=0 .
$$

Suppose $f(\bar{x}) \neq 0$. By (9), it is easy to see that

$$
2 \bar{x}^{T} P\left[-A \bar{x}+\left(W_{0}+W_{1}\right) f(\bar{x})\right]=0
$$

and

$$
2 f(\bar{x})^{T} S\left[-A \bar{x}+\left(W_{0}+W_{1}\right) f(\bar{x})\right]=0 .
$$

Note that

$$
\begin{aligned}
& 2 f(\bar{x})^{T} S\left[-A \bar{x}+\left(W_{0}+W_{1}\right) f(\bar{x})\right] \\
& \quad \leq f(\bar{x})^{T} K f(\bar{x})+2 f(\bar{x})^{T} H f(\bar{x}) \\
& \quad \leq f(\bar{x})^{T} K f(\bar{x})+2 \bar{x}^{T} H \Sigma f(\bar{x})
\end{aligned}
$$

where

$$
K=-2 S A \Sigma^{-1}+S\left(W_{0}+W_{1}\right)+\left(W_{0}+W_{1}\right)^{T} S-2 H .
$$

Then, it follows from (10)-(12) that

$$
\begin{aligned}
2 \bar{x}^{T} P\left[-A \bar{x}+\left(W_{0}+W_{1}\right) f(\bar{x})\right]+ & 2 \bar{x}^{T} H \Sigma f(\bar{x}) \\
& +f(\bar{x})^{T} K f(\bar{x}) \geq 0 .
\end{aligned}
$$

Now, applying Lemma 1, we have

$2 \bar{x}^{T}\left[P\left(W_{0}+W_{1}\right)+H \Sigma\right] f(\bar{x})-\bar{x}^{T}(P A+A P) \bar{x} \leq f(\bar{x})^{T} J f(\bar{x})$ where

$J=\left[P\left(W_{0}+W_{1}\right)+H \Sigma\right]^{T}(P A+A P)^{-1}\left[P\left(W_{0}+W_{1}\right)+H \Sigma\right]$.

This together with (13) implies

$$
f(\bar{x})^{T}(J+K) f(\bar{x}) \geq 0 .
$$

On the other hand, pre- and post-multiplying (6) by

$$
\left[\begin{array}{cccc}
I & 0 & 0 & 0 \\
0 & I & I & W_{1}^{T}
\end{array}\right]
$$


and its transpose, respectively, we obtain

$$
\left[\begin{array}{cc}
-P A-A P & P\left(W_{0}+W_{1}\right)+H \Sigma \\
\left(W_{0}+W_{1}\right)^{T} P+\Sigma H & K
\end{array}\right]<0
$$

which, by the Schur complement formula, results in

$$
J+K<0 .
$$

This contradicts with (14). Therefore, we have that at the equilibrium point $f(\bar{x})=0$, which, by (9), implies $\bar{x}=0$. That is, the origin of the delayed $\mathrm{CNN}$ in (4) is the unique solution to (9), and hence, (4) has a unique equilibrium point.

Next, we show that the unique equilibrium point of (4) is globally asymptotically stable. To this end, we denote

$$
x_{t}=x(t+\theta), \quad-\tau \leq \theta \leq 0
$$

and

$$
S=\operatorname{diag}\left(s_{1}, s_{2}, \ldots, s_{n}\right)
$$

Define a Lyapunov-Krasovskii functional candidate for system (4) as

$$
V\left(x_{t}\right)=V_{1}\left(x_{t}\right)+V_{2}\left(x_{t}\right)+V_{3}\left(x_{t}\right)
$$

where

$$
\begin{aligned}
& V_{1}\left(x_{t}\right)=x(t)^{T} P x(t) \\
& V_{2}\left(x_{t}\right)=2 \sum_{i=1}^{n} s_{i} \int_{0}^{x_{i}(t)} f_{i}(\alpha) d \alpha \\
& V_{3}\left(x_{t}\right)=\int_{t-\tau}^{t} f(x(\alpha))^{T} Q f(x(\alpha)) d \alpha .
\end{aligned}
$$

Then, the time derivative of $V\left(x_{t}\right)$ along the solution of (4) gives

$$
\begin{aligned}
\dot{V}\left(x_{t}\right)= & 2 x(t)^{T} P\left[-A x(t)+W_{0} f(x(t))+W_{1} f(x(t-\tau))\right] \\
& +2 f(x(t))^{T} S\left[-A x(t)+W_{0} f(x(t))+W_{1} f(x(t-\tau))\right] \\
& +f(x(t))^{T} Q f(x(t))-f(x(t-\tau))^{T} Q f(x(t-\tau)) .
\end{aligned}
$$

Considering the relationship in (5) and noting that $S>0$ and $H>0$ are diagonal matrices, we can deduce

$$
\begin{aligned}
& -2 f(x(t))^{T} S A x(t) \leq-2 f(x(t))^{T} S A \Sigma^{-1} f(x(t)) \\
& f(x(t))^{T} H f(x(t)) \leq x(t)^{T} H \Sigma f(x(t)) .
\end{aligned}
$$

Noting $Z>0$ and using Lemma 1, we have

$$
\begin{gathered}
2 f(x(t))^{T} S W_{1} f(x(t-\tau)) \leq f(x(t))^{T} S Z^{-1} S f(x(t)) \\
+f(x(t-\tau))^{T} W_{1}^{T} Z W_{1} f(x(t-\tau)) .
\end{gathered}
$$

Let

$$
\Omega=Q-2 S A \Sigma^{-1}+S W_{0}+W_{0}^{T} S+S Z^{-1} S .
$$

Then, by (18)-(21), it can be shown that

$$
\begin{aligned}
\dot{V}\left(x_{t}\right) \leq & -2 x(t)^{T} P A x(t)+2 x(t)^{T}\left(P W_{0}+H \Sigma\right) f(x(t)) \\
& +2 x(t)^{T} P W_{1} f(x(t-\tau)) \\
& -f(x(t))^{T}(2 H-\Omega) f(x(t)) \\
& +f(x(t-\tau))^{T}\left(W_{1}^{T} Z W_{1}-Q\right) f(x(t-\tau)) .
\end{aligned}
$$

From the inequality in (6), it is easy to see that

$$
2 H-\Omega>0
$$

Therefore, by using Lemma 1 again, we obtain

$$
\begin{aligned}
& 2 x(t)^{T}\left(P W_{0}+H \Sigma\right) f(x(t))-f(x(t))^{T}(2 H-\Omega) f(x(t)) \\
& \leq x(t)^{T}\left(P W_{0}+H \Sigma\right)(2 H-\Omega)^{-1}\left(P W_{0}+H \Sigma\right)^{T} x(t) .
\end{aligned}
$$

This together with (22) gives

$$
\dot{V}\left(x_{t}\right) \leq \eta(t)^{T} \Pi \eta(t)
$$

where

$$
\begin{aligned}
\eta(t) & =\left[\begin{array}{ll}
x(t)^{T} & f(x(t-\tau))^{T}
\end{array}\right]^{T} \\
\Pi & =\left[\begin{array}{cc}
\Theta & P W_{1} \\
W_{1}^{T} P & W_{1}^{T} Z W_{1}-Q
\end{array}\right] \\
\Theta & =-P A-A P+\left(P W_{0}+H \Sigma\right)(2 H-\Omega)^{-1}\left(P W_{0}+H \Sigma\right)^{T} .
\end{aligned}
$$

Now, applying the Schur complement formula to (6) gives that $\Pi<0$. Therefore

$$
\dot{V}\left(x_{t}\right) \leq-a\|x(t)\|^{2}
$$

where

$$
a=\lambda_{\min }(-\Pi)>0 .
$$

Finally, by [12], it follows from (23) that the time-delay system $(\Sigma)$ is asymptotically stable. This completes the proof.

Remark 1: The Lyapunov-Krasovskii functional used in Theorem 1 is more general than that in [1], which will result in the fact that Theorem 1 will be less conservative than the condition derived in [1]. It is also worth pointing out that Theorem 1 is obtained under the assumption that the activation functions are nonstrict, while [13, Theorem 2] is obtained under the assumption that the activation functions are strict. Therefore, Theorem 1 in this paper is applicable to a wider class of delayed CNNs than [13, Theorem 2].

Next, we provide an example to illustrate the reduced conservatism of Theorem 1 by comparing it with recently reported delay-independent asymptotic stability results in the literature.

Example 1: Consider a delayed continuous-time CNN in (4) with parameters

$$
\begin{aligned}
A= & {\left[\begin{array}{ccc}
0.1456 & 0 & 0 \\
0 & 1.9886 & 0 \\
0 & 0 & 1.4947
\end{array}\right] } \\
W_{0}= & {\left[\begin{array}{ccc}
-0.0308 & 1.4766 & -1.3099 \\
0.9884 & -0.8138 & -0.8674 \\
-0.5990 & 0.6450 & -0.4742
\end{array}\right] } \\
W_{1}= & {\left[\begin{array}{ccc}
0.2669 & -0.4936 & -0.1156 \\
2.2456 & 0.6135 & 0.5350 \\
0.1320 & -1.4389 & -0.3550
\end{array}\right] . }
\end{aligned}
$$

We assume that

$$
\sigma_{1}=0.1680, \quad \sigma_{2}=0.1795, \quad \sigma_{3}=0.3876 .
$$

For this delayed CNN, it can be checked that the matrix inequalities in both [1, Theorem 1] and [13, Theorem 2] have no solutions. Also, it is found that the asymptotic stability conditions in [6] and [18] are not satisfied. Therefore, Theorem 1 in [1], Theorem 2 in [13], and the results in [6] and [18] fail to conclude 
whether this delayed CNN is asymptotically stable or not. However, by resorting to the Matlab LMI Control Toolbox, we find that the LMI in Theorem 1 in this paper is feasible. Hence, by Theorem 1, it can be seen that the delayed CNN is asymptotic stable. This shows that the condition given in Theorem 1 is less conservative than those in [1], [6], [13], and [18].

Considering delay-dependent asymptotic stability conditions are generally less conservative than delay-independent ones, we now provide such a condition for the delayed CNN (4) in the following theorem.

Theorem 2: The origin of the delayed CNN in (4) is the unique equilibrium point and it is globally asymptotically stable for any delay $0<\tau \leq \bar{\tau}$ if there exist matrices $P>0, Q>0$, $Z>0, \Phi>0$ and three diagonal matrices $S>0, H>0$ and $Y>0$ such that the following LMI holds:

$$
\left[\begin{array}{ccc}
-P A-A P+\bar{\tau} A \Phi A & P W_{1}-\bar{\tau} A \Phi W_{1}+Y & 0 \\
W_{1}^{T} P-\bar{\tau} W_{1}^{T} \Phi A+Y & \Gamma & -\bar{\tau} Y \\
0 & -\bar{\tau} Y & -\bar{\tau} \Phi \\
W_{0}^{T} P+\Sigma H-\bar{\tau} W_{0}^{T} \Phi A & 0 & 0 \\
0 & 0 & 0 \\
P W_{0}+H \Sigma-\bar{\tau} A \Phi W_{0} & 0 \\
0 & 0 \\
0 & 0 \\
\Omega-2 H & S+\bar{\tau} W_{0}^{T} \Phi \\
S+\bar{\tau} \Phi W_{0} & -Z
\end{array}\right]<0
$$

where $\Sigma$ is given in (7), and

$$
\begin{aligned}
& \Gamma=W_{1}^{T}(Z+\bar{\tau} \Phi) W_{1}-Q-2 Y \Sigma^{-1} \\
& \tilde{\Omega}=Q-2 S A \Sigma^{-1}+S W_{0}+W_{0}^{T} S+\bar{\tau} W_{0}^{T} \Phi W_{0} .
\end{aligned}
$$

Proof: Under the condition of the theorem, the uniqueness of the equilibrium point can be established by following a similar line as in the proof of Theorem 1, and thus is omitted. Now, we prove that the unique equilibrium point of (4) is globally asymptotically stable. To this end, we define a Lyapunov-Krasovskii functional candidate for (4) as

$$
\tilde{V}\left(x_{t}\right)=V_{1}\left(x_{t}\right)+V_{2}\left(x_{t}\right)+V_{3}\left(x_{t}\right)+V_{4}\left(x_{t}\right)
$$

where $V_{1}\left(x_{t}\right), V_{2}\left(x_{t}\right)$ and $V_{3}\left(x_{t}\right)$ are defined in (15)-(17), respectively, and

$$
V_{4}\left(x_{t}\right)=\int_{-\tau}^{0} \int_{t+\beta}^{t} \dot{x}(\alpha)^{T} \Phi \dot{x}(\alpha) d \alpha d \beta .
$$

Then, the time-derivative of $V_{4}\left(x_{t}\right)$ along the solution of (4) provides

$$
\begin{aligned}
\dot{V}_{4}\left(x_{t}\right) \leq \frac{1}{\tau} \int_{t-\tau}^{t}\{ & \bar{\tau}\left[-A x(t)+W_{0} f(x(t))+W_{1} f(x(t-\tau))\right]^{T} \\
& \times \Phi\left[-A x(t)+W_{0} f(x(t))+W_{1} f(x(t-\tau))\right]^{T} \\
& -2 \tau f(x(t-\tau))^{T} Y \dot{x}(\beta) \\
& +2 f(x(t-\tau))^{T} Y x(t) \\
& -2 f(x(t-\tau))^{T} Y x(t-\tau) \\
& \left.-\tau \dot{x}(\beta)^{T} \Phi \dot{x}(\beta)\right\} d \beta
\end{aligned}
$$

Noting that $Y>0$ is a diagonal matrix, we then have

$-f(x(t-\tau))^{T} Y x(t-\tau) \leq-f(x(t-\tau))^{T} Y \Sigma^{-1} f(x(t-\tau))$.

Considering (18), (19), (25) and (26), it can be deduced that

$$
\begin{aligned}
\dot{\tilde{V}}\left(x_{t}\right) \leq \frac{1}{\tau} \int_{t-\tau}^{t}[ & x(t)^{T}(\bar{\tau} A \Phi A-P A-A P) x(t) \\
& +2 x(t)^{T}(P-\bar{\tau} A \Phi) W_{0} f(x(t)) \\
& +2 x(t)^{T}\left(P W_{1}-\bar{\tau} A \Phi W_{1}+Y\right) f(x(t-\tau)) \\
& +f(x(t))^{T} \tilde{\Omega} f(x(t)) \\
& +f(x(t))^{T}\left(S+\bar{\tau} W_{0}^{T} \Phi\right) W_{1} f(x(t-\tau)) \\
& +f(x(t-\tau))^{T}\left(\bar{\tau} W_{1}^{T} \Phi W_{1}-Q-2 Y \Sigma^{-1}\right) \\
& \times f(x(t-\tau))-2 \tau f(x(t-\tau))^{T} Y \dot{x}(\beta) \\
& \left.-\tau \dot{x}(\beta)^{T} \Phi \dot{x}(\beta)\right] d \beta .
\end{aligned}
$$

Now, by Lemma 1, it can be shown that

$$
\begin{aligned}
& 2 f(x(t))^{T}\left(S+\bar{\tau} W_{0}^{T} \Phi\right) W_{1} f(x(t-\tau)) \\
& \quad \leq f(x(t))^{T}\left(S+\bar{\tau} W_{0}^{T} \Phi\right) Z^{-1}\left(S+\bar{\tau} W_{0}^{T} \Phi\right) f(x(t)) \\
& \quad+f(x(t-\tau))^{T} W_{1}^{T} Z W_{1} f(x(t-\tau)) .
\end{aligned}
$$

Then, it follows from (20), (27), and (28) that

$$
\begin{aligned}
\dot{\tilde{V}}\left(x_{t}\right) \leq \frac{1}{\tau} \int_{t-\tau}^{t}[ & x(t)^{T}(\bar{\tau} A \Phi A-P A-A P) x(t) \\
& +2 x(t)^{T}\left(P W_{0}-\bar{\tau} A \Phi W_{0}+H \Sigma\right) f(x(t)) \\
& +2 x(t)^{T}\left(P W_{1}-\bar{\tau} A \Phi W_{1}+Y\right) f(x(t-\tau)) \\
& -f(x(t))^{T}(2 H-\hat{\Omega}) f(x(t)) \\
& +f(x(t-\tau))^{T} \Gamma f(x(t-\tau)) \\
& -2 \tau f(x(t-\tau))^{T} Y \dot{x}(\beta) \\
& \left.-\tau \dot{x}(\beta)^{T} \Phi \dot{x}(\beta)\right] d \beta
\end{aligned}
$$

where

$$
\hat{\Omega}=\tilde{\Omega}+\left(S+\bar{\tau} W_{0}^{T} \Phi\right) Z^{-1}\left(S+\bar{\tau} W_{0}^{T} \Phi\right) .
$$

By (24), it is easy to have that

$$
2 H-\hat{\Omega}>0 .
$$

Then, by Lemma 1, it can be deduced that

$$
\begin{aligned}
2 x(t)^{T} & \left(P W_{0}-\bar{\tau} A \Phi W_{0}+H \Sigma\right) f(x(t)) \\
- & f(x(t))^{T}(2 H-\hat{\Omega}) f(x(t)) \\
\leq & x(t)^{T}\left(P W_{0}-\bar{\tau} A \Phi W_{0}+H \Sigma\right) \\
\quad & \times(2 H-\hat{\Omega})^{-1}\left(P W_{0}-\bar{\tau} A \Phi W_{0}+H \Sigma\right)^{T} x(t) .
\end{aligned}
$$

This together with (29) gives

$$
\dot{\tilde{V}}\left(x_{t}\right) \leq \frac{1}{\tau} \int_{t-\tau}^{t} \xi(t, \beta)^{T} \Upsilon(\tau) \xi(t, \beta) d \beta
$$


where

$$
\begin{aligned}
& \Lambda=\bar{\tau} A \Phi A-P A-A P+\left(P W_{0}-\bar{\tau} A \Phi W_{0}+H \Sigma\right) \\
& \times(2 H-\hat{\Omega})^{-1}\left(P W_{0}-\bar{\tau} A \Phi W_{0}+H \Sigma\right)^{T} \\
& \Upsilon(\tau)=\left[\begin{array}{ccc}
\Lambda & P W_{1}-\bar{\tau} A \Phi W_{1}+Y & 0 \\
W_{1}^{T} P-\bar{\tau} W_{1}^{T} \Phi A+Y & \Gamma & -\tau Y \\
0 & -\tau Y & -\tau \Phi
\end{array}\right] \\
& \xi(t, \beta)=\left[\begin{array}{lll}
x(t)^{T} & f(x(t-\tau))^{T} \quad \dot{x}(\beta)^{T}
\end{array}\right]^{T} .
\end{aligned}
$$

Applying the Schur complement formula to (24), we have that for all $0<\tau \leq \bar{\tau}$

$$
\left[\begin{array}{cc}
\Lambda & P W_{1}-\bar{\tau} A \Phi W_{1}+Y \\
W_{1}^{T} P-\bar{\tau} W_{1}^{T} \Phi A+Y & \Gamma+\tau Y \Phi^{-1} Y
\end{array}\right]<0
$$

which, by the Schur complement formula implies that $\Upsilon(\tau)<$ 0 . Then

$$
\dot{V}\left(x_{t}\right) \leq-b\|x(t)\|^{2}
$$

where

$$
b=\lambda_{\min }(-\Upsilon(\tau))>0
$$

Therefore, we have that (4) is asymptotically stable for any delay $0<\tau \leq \bar{\tau}$.

The following examples are given to show the reduced conservatism of the delay-dependent asymptotic stability result in Theorem 2.

Example 2: Consider a delayed continuous-time CNN in (4) with parameters

$$
\begin{aligned}
A= & {\left[\begin{array}{cccc}
1.2769 & 0 & 0 & 0 \\
0 & 0.6231 & 0 & 0 \\
0 & 0 & 0.9230 & 0 \\
0 & 0 & 0 & 0.4480
\end{array}\right] } \\
W_{0}= & {\left[\begin{array}{cccc}
-0.0373 & 0.4852 & -0.3351 & 0.2336 \\
-1.6033 & 0.5988 & -0.3224 & 1.2352 \\
0.3394 & -0.0860 & -0.3824 & -0.5785 \\
-0.1311 & 0.3253 & -0.9534 & -0.5015
\end{array}\right] } \\
W_{1}= & {\left[\begin{array}{cccc}
0.8674 & -1.2405 & -0.5325 & 0.0220 \\
0.0474 & -0.9164 & 0.0360 & 0.9816 \\
1.8495 & 2.6117 & -0.3788 & 0.8428 \\
-2.0413 & 0.5179 & 1.1734 & -0.2775
\end{array}\right] . }
\end{aligned}
$$

In this example, we suppose that

$$
\begin{array}{ll}
\sigma_{1}=0.1137, & \sigma_{2}=0.1279 \\
\sigma_{3}=0.7994, & \sigma_{4}=0.2386 .
\end{array}
$$

For this delayed CNN, it can be checked that the asymptotic stability conditions in [6] and [18], Theorem 1 in [1], Theorem 2 in [13] as well as Theorem 1 in this paper are not satisfied. Therefore, they fail to conclude whether this delay system is asymptotically stable or not. Now, we resort to Theorem 2 in this paper to check the asymptotic stability of the delay system. It is found that the LMI in (24) is feasible for all delay $0<\tau \leq$ $\bar{\tau}=1.4224$. Therefore, by Theorem 2 , we conclude that the delay system is delay-dependent asymptotically stable. If we use
Theorem 1 in [17], we can calculate the largest allowed value of $\bar{\tau}=0.3518$, which is $75.27 \%$ smaller than that obtained by our method. This shows that the condition given in Theorem 2 is less conservative than that in [17] when checking the asymptotic stability of a given delay neural network.

\section{CONCLUSION}

This paper has provided improved criteria for the existence of a unique equilibrium point and its global asymptotic stability of continuous delayed CNNs. Both delay-dependent and delay-independent stability conditions have been proposed in terms of LMIs, which can be checked easily by using recently developed standard algorithms. Examples via numerical comparisons with the recently reported results have demonstrated the lower conservatism of the proposed criteria in this paper.

\section{REFERENCES}

[1] S. Arik, "Global asymptotic stability of a larger class of neural networks with contant time delay," Phys. Lett. A, vol. 311, pp. 504-511, 2003.

[2] S. Arik and V. Tavsanoglu, "Equilibrium analysis of delayed CNNs," IEEE Trans Circuits Syst. I, Fundam. Theory Appl., vol. 45, no. 1, pp. 168-171, Jan. 1998.

[3] P. Baldi and A. Atiya, "How delays affect neural dynamics and learning," IEEE Trans. Neural Netw., vol. 5, no. 6, pp. 612-621, Dec. 1994

[4] S. Boyd, L. El Ghaoui, E. Feron, and V. Balakrishnan, Linear Matrix Inequalities in System and Control Theory. Philadelphia, PA: SIAM, 1994

[5] J. Cao, "Global stability conditions for delayed CNNs," IEEE Trans Circuits Syst. I, Fundam. Theory Appl., vol. 48, no. 12, pp. 1330-1333, Dec. 2001.

[6] J. Cao and D. Zhou, "Stability analysis of delayed cellular neural networks," Neural Netw., vol. 11, pp. 1601-1605, 1998.

[7] L. O. Chua and L. Yang, "Cellular neural networks: applications," IEEE Trans. Circuits Syst., vol. 35, no. 12, pp. 1273-1290, Dec. 1988.

[8] _ _ "Cellular neural networks: theory," IEEE Trans. Circuits Syst., vol. 35, no. 12, pp. 1257-1272, Dec. 1988.

[9] P. P. Civalleri, M. Gilli, and L. Pandolfi, "On stability of cellular neura networks with delay," IEEE Trans Circuits Syst. I, Fundam. Theory Appl., vol. 40, no. 2, pp. 157-165, Feb. 1993.

[10] M. Forti and A. Tesi, "New conditions for global stability of neural networks with application to linear and quadratic programming problems," IEEE Trans Circuits Syst. I, Fundam. Theory Appl., vol. 42, no. 3, pp. 354-365, Mar. 1995.

[11] T. Kohonen, Self-Organizing Maps. Berlin, Germany: SpringerVerlag, 2001.

[12] V. B. Kolmanovskii and A. D. Myshkis, Introduction to the Theory and Applications of Functional Differential Equations. Dordrecht, The Netherlands: Kluwer, 1999

[13] X. Liao, G. Chen, and E. N. Sanchez, "LMI-based approach for asymptotically stability analysis of delayed neural networks," IEEE Trans Circuits Syst. I, Fundam. Theory Appl., vol. 49, no. 10, pp. 1033-1039, Oct 2002

[14] A. N. Michel, J. A. Farrel, and W. Porod, "Qualitative analysis of neural networks," IEEE Trans. Circuits Syst., vol. 36, no. 3, pp. 229-243, Mar 1989

[15] S. Xu, T. Chen, and J. Lam, "Robust $H_{\infty}$ filtering for uncertain Markovian jump systems with mode-dependent time delays," IEEE Trans. Autom. Contr., vol. 48, no. 8, pp. 900-907, Aug. 2003.

[16] S. Xu, J. Lam, and C. Yang, "Robust stability analysis and stabilization for uncertain linear neutral delay systems," Int. J. Syst. Sci., vol. 33, pp 1195-1206, 2002.

[17] H. Ye, A. N. Michel, and K. Wang, "Global stability and local stability of Hopfield neural networks with delays," Phys. Rev. E, vol. 50, pp. 4206-4213, 1994.

[18] Q. Zhang, R. Ma, and J. Xu, "Stability of cellular neural networks with delay," Electron. Lett., vol. 37, pp. 575-576, 2001. 